\title{
Measuring Passenger Car Equivalents (PCE) for Heavy Vehicle on Two Lane Highway Segments Operating Under Various Traffic Conditions
}

\author{
Pan Lu $\mathbb{D}^{1},{ }^{1}$ Zijian Zheng, ${ }^{2}$ Denver Tolliver, ${ }^{3}$ and Danguang Pan $\mathbb{D}^{4}$ \\ ${ }^{1}$ Associate Professor/Associate Research Fellow, Department of Transportation, Logistics, and Finance, NDSU Dept 2880, \\ P.O. Box 6050, Fargo, ND 58108-6050, USA \\ ${ }_{2}^{2}$ International Supply Chain \& Logistics Analyst, Gates Corporation, 1144 Fifteenth St. Suite 1400, Denver, CO 80202, USA \\ ${ }^{3}$ Director and Senior Research Fellow, Upper Great Plains Transportation institute (UGPTI), NDSU Dept 2880, P.O. Box 6050, \\ Fargo, ND 58108-6050, USA \\ ${ }^{4}$ Professor, Department of Civil Engineering, University of Science and Technology Beijing, No. 30 Xueyuan Ave. Haidian District, \\ Beijing 110001, China \\ Correspondence should be addressed to Pan Lu; pan.lu@ndsu.edu
}

Received 22 October 2018; Revised 9 January 2019; Accepted 21 November 2019; Published 17 January 2020

Academic Editor: Zhi-Chun Li

Copyright (C) 2020 Pan Lu et al. This is an open access article distributed under the Creative Commons Attribution License, which permits unrestricted use, distribution, and reproduction in any medium, provided the original work is properly cited.

\begin{abstract}
Passenger Car Equivalent (PCE) is essential for transportation engineering to assess heavy vehicles' (HV) impact on highway operations and capacity planning. Highway Capacity Manual 2010 (HCM 2010) used PCE values and percent of heavy vehicles to account the impacts on both highway planning and operation, however, PCE values in the latest version of HCM derived based on the steady and balanced two-lane-two-way (TLTW) traffic flows. The objective of the study is to identify PCE values for TLTW highway at various traffic volume with an emphasis on congestion conditions. This study introduces an analytical model, combining a headway-based and a delay-based algorithms, for estimating PCEs of HV on a TLTW highway. This study contributes to the literature by providing relationships among PCE, the traffic volume level (TVL) of both lanes, and the TVL duration on a TLTW highway. Traffic volume was categorized into five levels: TVL A ( $<250 \mathrm{pc} / \mathrm{h})$, TVL B $(250-375 \mathrm{pc} / \mathrm{h})$, TVL C (375-600 pc/h), TVL D $(600-850 \mathrm{pc} / \mathrm{h})$, and TVL E $(>850 \mathrm{pc} / \mathrm{h})$. The results indicate that on a TLTW highway, the TVLs of both lanes and their durations have significant impact on PCE values. In general, PCE values increase as TVL duration increases. Trucks have much higher impacts on operation under unbalanced conditions of TVL A with D, TVL B with C, and TVL D with B, when duration time is greater than one hour. When both lanes are saturated, trucks' effect on capacity diminishes over time, and PCE values are approaching to 1.0.
\end{abstract}

\section{Introduction and Literature Review}

Because of the recent oil boom in Western North Dakota, the number of large oil trucks on two-lane rural highways has increased dramatically to transport oil-related products. The sudden surge in the number of large trucks results in severe traffic issues related to highway design. Trucks consume more highway capacity and have a greater impact on following traffic than do passenger cars. Thus, it is critical to measure such differences to help the Department of Transportation (DOT) and other agencies with highway design and congestion challenges.

The passenger car equivalent (PCE) factor is commonly used to assess differences between large trucks and passenger cars in terms of highway capacity and congestion analysis [1]. PCE factor measures the magnitude of large vehicles' effect on following traffic compared to passenger cars [2]. Previous researchers developed numerous models to measure PCE factors, such as headway-based method [3], delay-based method $[4,5]$, average-travel speed-based method [6-9], truck-percentage-based method [10], density-based method [11], and time-spent-on-following-based method [12]. These models improve the understanding of the mechanisms of PCE and help agencies to determine PCEs. However, the models all have encountered some limitations.

There are numerous studies focused on PCE factor. However, the amount of research focusing on two-lane 
highway is limited. HCM 1965 [6] introduced a method to assess two-lane highway PCE values. It proposed an averagespeed-based PCE model. This method is rather straight forward, however, it assumes opposing-traffic had no effect on passing, which is not always true. The method provided a useful result for two-lane highway, but is not appropriate for use when the opposite lane is congested. HCM 2000 [12] studied the relationship between traffic flow and PCE values, however, it assumes balanced and steady traffic flows on two-lane highways (TLTW) which is unrealistic in most of the cases. Al-Kaisy, Jung, and Rakha pointed out that roadway level of congestion is a significant variable affecting truck PCEs on a freeway [13].

Intuitively thinking, for TLTW highways, traffic volumes of both lanes should have effects on PCE values to reflect unbalanced traffic impacts. The effect of large trucks on upstream traffic can be measured by queue length caused by the large trucks [14]. The queue length is determined by a queue birth rate and death rate. On a TLTW highway, passing can be taken only when there is a long enough gap in oncoming traffic, and opportunities to pass are determined by traffic volume in the opposite lane. In addition, traffic volume of a studied lane determines the birth rate of a queue. Thus, while either lane's traffic volume varies, PCE value is expected to change too.

Truck length and weight causes them to be hard to maneuver. In addition to their slower deceleration rate, trucks need longer distances to stop compared to passenger-cars with traveling at the same speed. Thus, trucks typically maintain a longer following distance behind the vehicles in front of them compared to passenger cars. Therefore, trucks have impacts on both adjacent vehicles and upstream traffic coming toward them.

In HCM 1985 [15], the authors suggested measuring PCE value based on "percent time delay," which is the proportion of the cumulative travel time that a driver spends following other vehicles to the entire travel time. Another element of the study was that the capacity of a two-lane highway was proven to be a function of the directional split of traffic. Archilla questioned HCM 1985 results and stated that the directional split factors appeared to be overestimated [15]. Archilla conducted an uneven directional split factor analysis, in which better operational performance was observed than in the 50/50 split, which was considered as an ideal condition by HCM 1985 and was supposed to obtain the best operational performance [15]. Craus et al. [16] continually improved the HCM 1965 method, and developed another model for trucks on a two-lane highway by computing the time that following vehicles needed to pass a truck and a passenger-car. In their study, they assumed that headways between vehicles are distributed exponentially. However, this assumption is only valid when traffic volume is low, and traffic arrival follows Poisson distribution [16]. HCM 2010 as the latest version of highway capacity manual [17] developed two sets of PCE values for a two-lane highway: one set for average travel speed and another set for percent time spent following. This method try to take into considerations of both average travel speed and delay however, it assumes that trucks and passenger cars travel at the same average speed.
Another factor not considered in previous studies is the duration of traffic volume of each lane. As traffic volume is associated with the queue growth rate, the duration impacts the queue length as well. For example, when both of lanes are saturated, queue length grows as a large number of vehicles join the queue. As long as the highway maintains at such traffic volume, the queue length will keep growing. Meanwhile, PCE values determined by the queue length are a function of the traffic volume duration.

Based on the discussion above, a new analytical model, combining a headway-based and delay-based algorithms, is proposed in this study to calculate PCE factors for HVs considering various TVLs and their durations in both lanes for a TLTW highway. The developed new sets of PCE values will be presented.

\section{Methodology}

2.1. Introduction. As discussed above, previous studies did not consider two factors' impact on truck PCEs on a two-lane highway: (1) traffic volume of each lane, and (2) duration of the traffic volume of each lane.

According to HCM 2010 model, mathematically, the PCE factor is to measure how much more impact one truck has on traffic flow, either measured by average travel speed or percent time spent following. In this study, to quantify the effect of trucks on traffic flow, an analytical model is proposed to calculate the PCE factor by comparing expected values of delay caused by a truck and a passenger-car. Expected value of length of traffic affected by slow vehicles is taken as the measurement of delay. In other words, the PCE factor is computed as the ratio of expected value of length of traffic que resulting from a truck to that resulting from a passenger-car. As shown in Figure 1, there is one slow-moving vehicle (a truck or a passenger car) in each studied platoon. The total traffic length resulting from the slow vehicle consists of two parts: (1) affected queue length resulting from the slow vehicle, introduced in detail in section on the queue length model; (2) distance between the two passenger cars adjacent to the slow vehicle, expressed in section on headway model. PCE value is mathematically expressed as Equation (1):

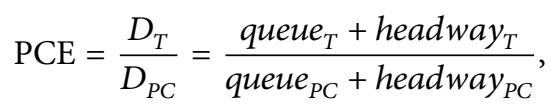

where

$D_{T}$ is expected value of delay caused by a truck.

$D_{P C}$ is expected value of delay caused by one slow passenger car with the same traveling speed as the slow truck.

headway $y_{T}$ is space between the passenger-car in front of the truck and the one following right behind the truck.

headway ${ }_{P C}$ is space between the passenger-car in front of the slow passenger car and the one following right behind it. $q^{q u e u e_{T}}$ is the length of queue caused by the truck.

queue $_{P C}$ is the length of queue caused by the slow passenger car. 

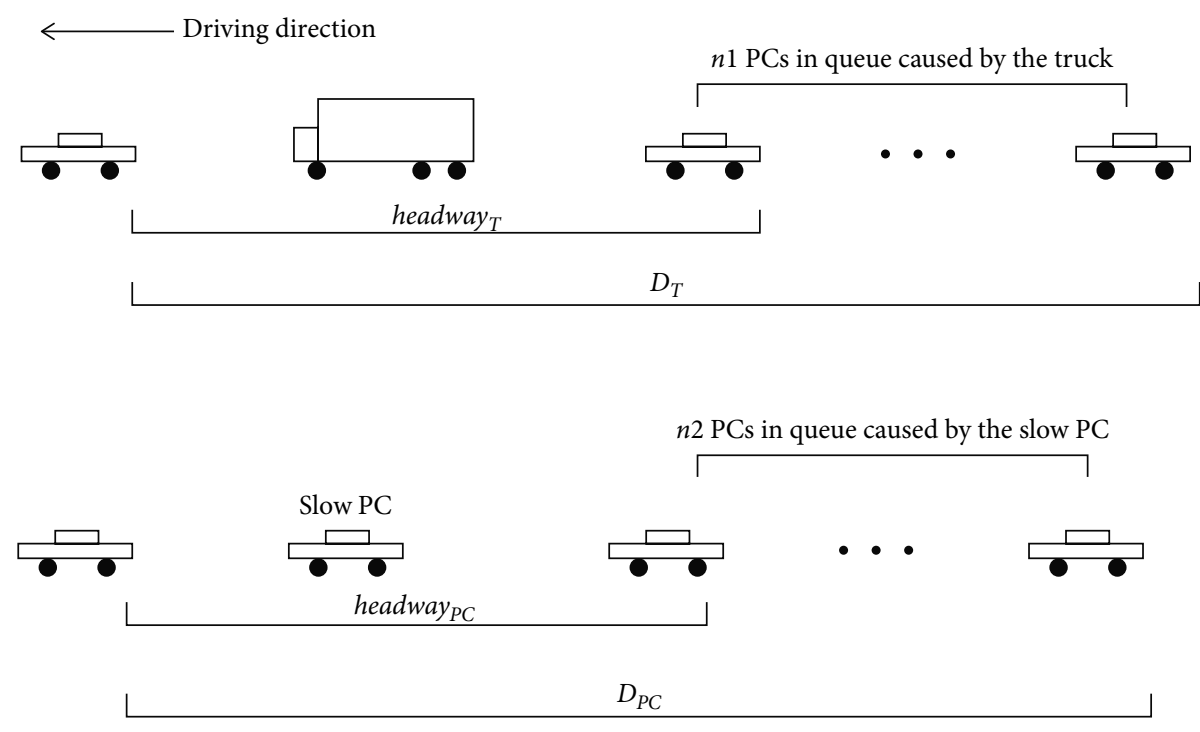

FIGURE 1: PCE factor model introduction.

\subsection{Queue Length Model}

2.2.1. Assumptions. To calculate queue ${ }_{T}$ and queue ${ }_{P C}$, some assumptions are made:

(1) This model focuses on one truck category operating on a two-lane rural highway in level terrain.

(2) No passing-lane is considered.

(3) Studied traffic platoon only consists of passenger cars and one truck.

(4) Only two vehicles get involved in the process of overtaking, which means no more than one vehicle can make a passing maneuver at a time.

A queue generated only when a vehicle follows another vehicle. According to HCM 2010, traffic volume affects following time that a vehicle spends on following another. Based on traffic volume (veh/h), the queue length model will be introduced under five levels: (A) $<250 \mathrm{pc} / \mathrm{h}$, (B) $250-375 \mathrm{pc} / \mathrm{h}$, (C) 375-600 pc/h, (D) 600-850 pc/h, and (E) $>850 \mathrm{pc} / \mathrm{h}$, where percent time-spend-following is $<20 \%, 20-40 \%, 40-60 \%$, $60-80 \%$, and $>80 \%$, respectively [17]. In the meantime, among different levels, traffic performance and headway distribution are different [3].

2.2.2. Under Level $A$ and B. According to Wolfgang, Louis, and William [18], in group (1), because of a low traffic flow rate, headway distribution and vehicle arrival distribution can be viewed as truly random, and are described by negative exponential distribution and Poisson distribution respectively. If the slow vehicle is considered as a service channel, upstream vehicles passing the slow vehicle can be considered as vehicles passing the service channel but with a relative speed in Equation (2). In this case, the queue length caused by the service channel or the slow vehicle is calculated in following step based on an $\mathrm{M} / \mathrm{M} / 1$ model [18].

To calculate queue length resulting from a truck or a slow passenger car, the truck and the slow passenger car are treated as service channels with their own speed $V_{T}$. When a truck and a slow passenger car are viewed as standing service channels, the rest of traffic (original speed denoted as $V_{C}$ ) in the same lane moves with a relative velocity [19]:

$$
V_{R}=V_{C}-V_{T} \text {. }
$$

Rate of arrival relates with traffic density and traffic velocity [20]. While considering the slow passenger as service channel, the traffic density $(K)$ remains the same, but, the relative average rate of arrival $\left(\lambda_{R}\right)$ would change to

$$
\lambda_{R}=V_{R} \times K=\left(V_{C}-V_{T}\right) \times K .
$$

Time spent by one passenger-car following slow vehicle is given by [5]:

$$
d_{1}=\left(\frac{1-e^{-\lambda \alpha}}{\lambda e^{-\lambda \alpha}}-\alpha\right) \cdot\left(\frac{u}{u+v}\right)
$$

where

$u$ is the speed of average of opposing traffic;

$v$ is the speed of the slow vehicle;

In $\mathrm{M} / \mathrm{M} / 1$ model, if upstream traffic arrival rate $\lambda_{R}$ is smaller than service rate $d_{1}$, average queue length $E_{m 1}$ can be calculated with Equation (5) [18]:

$$
E_{m 1}=\frac{\lambda_{R}^{2}}{d_{1}\left(d_{1}-\lambda_{R}\right)}
$$

where

$\lambda_{R}$ is arrival rate of upstream traffic calculated by Equation (3);

$d_{1}$ is calculated in Equation (4).

If upstream traffic arrival rate $\lambda_{R}$ is larger than service rate $d_{1}$, queue length will be a function of time and is calculated by Equation (6) [21] indicating how queue length grows with rate of $q$ over time:

$$
E\{Q(t)\}=E\{Q(t-\Delta t)\}+q \Delta t
$$




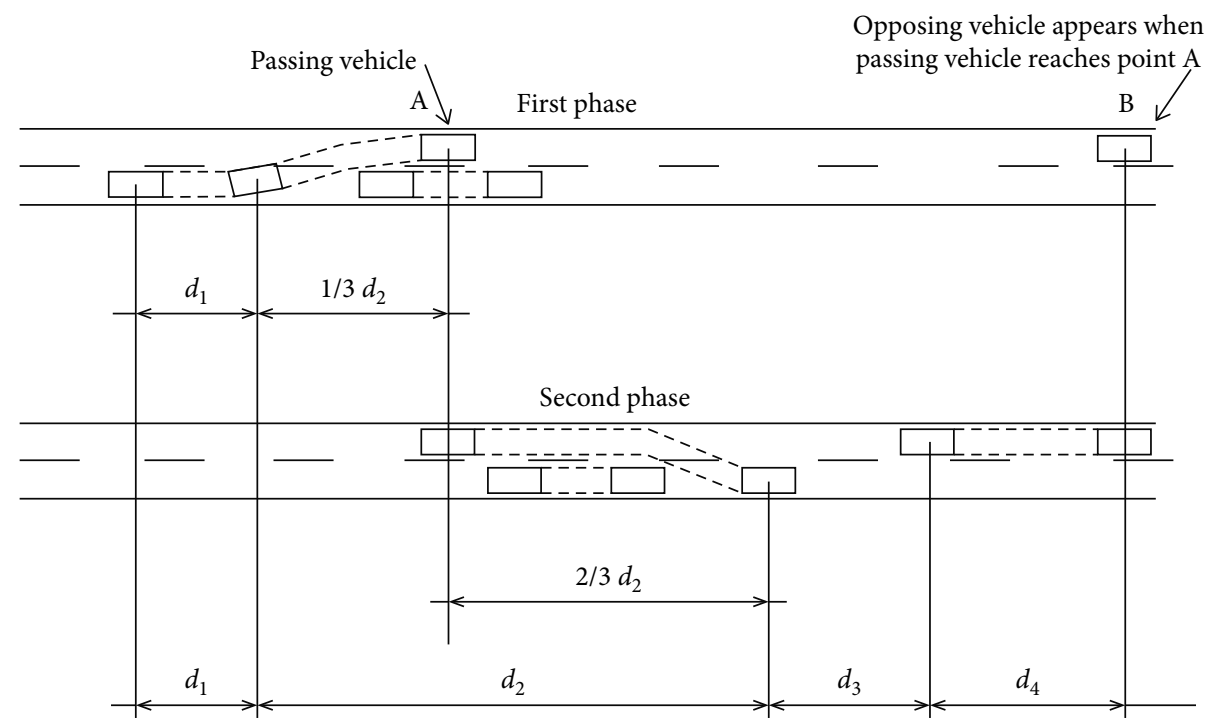

FIGURE 2: Diagram of passing sight distance components.

where $E\{Q(t)\}$ is queue length at time $t ; \Delta t$ is a small time period; $E\{Q(t-\Delta t)\}$ is queue length at time $t-\Delta t ; q$ is the coming traffic arrival rate in veh/h.

2.2.3. Under Level $C$ and $D$. Under this situation, traffic condition is more congested than under TVL A and B. Headway distribution in the opposing lane is no longer random. M3 headway distribution model is suggested for TVL C and D [22] to calculate possibility of a given headway:

$$
F(t)=(1-\theta) e^{-\lambda(t-\Delta)}, \quad t \geq 0
$$

where

$\lambda=$ flow rate in vehicle $/ s$

$\Delta=$ minimum headway between bunched vehicles;

$\theta=$ the proportion of constrained vehicles;

$t=$ headway;

$F(t)=$ probability distribution of headways.

With M3 headway distribution, the adjusted time that a passenger car follows a truck or a slow passenger car is stated in Equation (8) by combining Equations (7) and (4):

$$
d_{2}=\left(\frac{1-(1-\theta) e^{-\lambda(t-\Delta)}}{\lambda(1-\theta) e^{-\lambda(t-\Delta)}}-t\right) \cdot\left(\frac{u}{u+v}\right),
$$

where $d_{2}$ is the expected time that a passenger-car follows a slow vehicle. It can also be interpreted as the frequency that coming vehicles overtake the slow vehicle, which is the queue death rate.

Calculation of queue death rate is similar to the calculation under TVL A and B using the same model. Equation (6) is used to describe the relationship between queue length and time.

2.2.4. Under Level E. Under this situation, the highway reaches the capacity and headway between vehicles completely depends on vehicles rather than being randomly distributed. There is not enough space in the opposite lane to allow overtaking safely. Thus queue birth rate is equal to traffic arrival rate, which is independent of types of vehicle. Therefore, the delay caused by a truck and a slow passenger car is the same.

2.2.5. Passing Sight Distance. A minimal required passing sight distance model is shown in Figure 2 [23], where $d_{1}$ is perception-reaction-accelerate distance. At this distance, drivers will contemplate a passing maneuver and accelerate to the point of encroachment into the left lane. $d_{2}$ is the length of highway that is traversed by the passing vehicle, while it occupies the opposing lane. $d_{3}$ is the safety distance between passing vehicle and the vehicle from the opposing lane, when the passing vehicle returns back from the opposite lane. $d_{4}$ is the distance that the vehicle from the opposite lane travels while the passing vehicle travels the $2 / 3 d_{2}$.

2.3. Headway Model. In the studied platoon shown in Figure 1, calculation for headway ${ }_{T}$ and headway ${ }_{P C}$ is presented in this section. One difference between a truck and a passenger-car is their configuration. Overall length of an oil truck is 26 meters ( 85 feet), compared to the average length of a passenger car which is 4.2 meters (14 feet) [24]. Space between two vehicles for safety stopping purpose, often referred to as minimal headway $(\mathrm{MH})$ or safety distance $(\mathrm{SD})$, is another important criterion considered in the model.

Deceleration and weight differences between trucks and passenger-cars make SD different. When a passenger-car follows a truck, distance between them would be longer than when a passenger car follows another passenger car.

As shown in Figure 3, SD $S(t)$ is the distance between the two cars so that if the front vehicle brakes suddenly, the following vehicle can stop without colliding with the front one [18]. $T$ is the response time of the following driver who realizes the front vehicle is slowing down and he needs to hit the brake. Equation (9) is used to calculate safety distance [18]:

$$
S(t)=d_{1}+d_{2}+L-d_{3},
$$




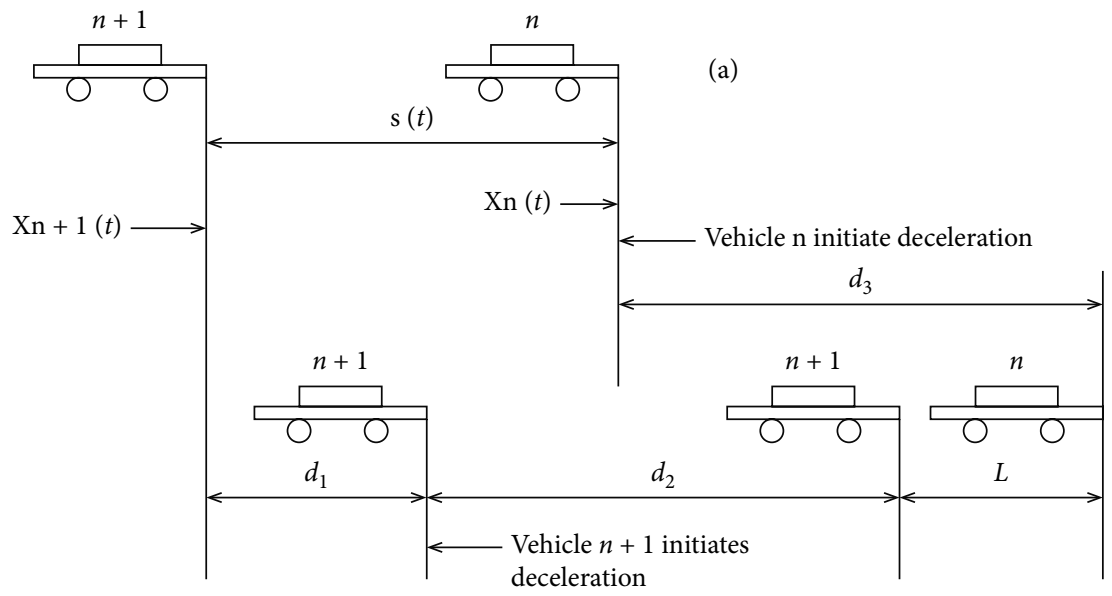

(b)

FIgURE 3: Safety distance in car following model.

where

$S(t)=$ at time $t$ headway between the leading vehicle and the following vehicle;

$d_{1}=$ during response time $T$, distance moved by the following vehicle;

$d_{2}=$ stopping distance for the following vehicle;

$d_{3}=$ stopping distance for the leading vehicle;

$L=$ safety distance between the two vehicles when they begin stopping.

2.4. PCE Factor. Combining the queue length model and the headway model, equations to calculate the value of the PCE factor are presented below, under three groups:

Group 1: under good travel condition with traffic flow rate less than $375 \mathrm{veh} / \mathrm{h}$, only a small number of passenger cars are affected by slow vehicles. Therefore, the queue will not expand infinitely. That is, as long as the travel condition remains consistent, and queue birth rate is lower than queue death rate, queue length will not exceed a fixed value [18]. In this case, PCE values will not change with time.

Group 2: infinite queue for both types of vehicles is possible when traffic flow rate is between $375 \mathrm{veh} / \mathrm{h}$ and $850 \mathrm{veh} / \mathrm{h}$. The PCE factor is related to time $(\Delta t)$ and is depicted in Equation (10)

$$
\begin{aligned}
\text { PCE } & =\frac{\text { Tlength }_{T}}{\text { Tlength }_{P C}}=\frac{\text { queue }_{T}+\text { head }_{\text {quey }}}{\text { que }_{P C}+\text { headway }_{P C}} \\
& =\frac{\left(\lambda_{R}-d_{2 T}\right) \cdot \Delta t+\text { head way }_{T}}{\left(\lambda_{R}-d_{2 P C}\right) \cdot \Delta t+\text { headway }_{P C}},
\end{aligned}
$$

where $d_{2 T}$ and $d_{2 P C}$ are the amount of time that a passenger-car spends following a truck and a slow passenger-car respectively. $\lambda_{R}$ is relative arrival rate with the slow vehicles as a reference. Arrival rate data is normally collected from a standing point. However, in the queue-length model, slow vehicles are selected as a reference, which is not a static point, but a moving reference. Therefore, relative arrival rate is used in the equation to calculate the value of the PCE factor.

Group 3: when a finite queue is formed for a passenger car and an infinite queue is formed for a truck, the PCE equation can be expressed by Equation (11):

$$
\begin{aligned}
\text { PCE } & =\frac{\text { Tlength }_{T}}{\text { Tlength }_{P C}}=\frac{\text { queue }_{T}+\text { headway }_{T}}{\text { queue }_{P C}+\text { head way }_{P C}} \\
& =\frac{\left(\lambda_{R}-d_{2 T}\right) \cdot \Delta t+\text { head way }_{T}}{\text { queue }_{P C}+\text { headway }_{P C}} .
\end{aligned}
$$

In this situation, the PCE factor value highly depends on the traffic volume duration $(\Delta t)$, because as time increases, the queue caused by a truck will continue accumulating, while the queue caused by a passenger car will remain consistent. As shown in Equation (11), all parameters are fixed values except for $\Delta t$. Therefore, the PCE value will increase as $\Delta t$ increases.

\section{Results Analysis}

PCE values are calculated and summarized in this section under various combinations of traffic volume levels (TVLs) of opposing traffic. In this section, "TVL $x$ with $y$ " denote the situation where the TVL of traveling lane is $x$, while the TVL of the opposing lane is $y$. Therefore, there are 25 combinations in total to be analyzed, based on five TVLs for each lane. It is demonstrated that the PCE value varies with different TVLs of both lanes, and also depends on the duration of the TVLs in both directions. Effects of TVL in opposing lane on PCE are selected to show the results in this research. Three types of relationships between PCE factor values and TVL duration were observed:

3.1. Two-Step Linear Relationship. When traffic condition is under TVL A with D, TVL B with C, and TVL D with A, PCE values and TVL duration have a two-step linear relationship, as shown in Figure 4. Under these situations, an infinite queue is generated for a slow truck. However, for a slow passenger car there are still opportunities to pass, and a finite queue is generated. Therefore, in this case, the PCE factor is still a function of time. Several interesting observations were discovered under these situations: (1) PCE increases as TVL duration increases which indicates that trucks will have increasingly more impact on traffic with congestion duration increasing when passing behavior is not an option for trucks, but is still possible for passenger cars. (2) PCE increases 


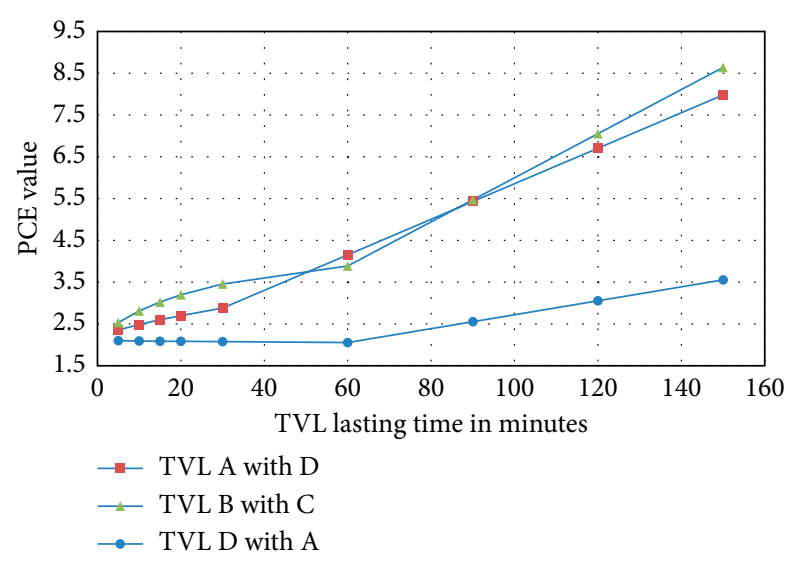

FIGURE 4: Linear relationship.

with TVL duration increasing at two linear rates. The TVL duration bench mark or inflection point for PCE increasing rate change varies from 30 minutes to one hour. The PCE value of TVL A with D has a half hour as a bench mark. With congestion duration in opposing lane increasing, but before it reaches a half hour, PCE value goes up with a relatively lower increasing rate from around 2 to 3 and from half hour and up, PCE value increases with relatively higher increasing rate to 4 at congestion, lasting about one hour. In the other two groups, the benchmark happens at congestion duration of about one hour instead of a half hour. (3) Trend and values of PCE under TVL A with D and TVL B with C are similar, and both of the two groups start and end at similar points. However, PCE values under TVL D with A are much lower than the other two groups, especially after the congestion condition lasts for a while. And (4) all three cases start off with PCE values around 2.2.

3.2. Negative Exponential Relationship. Under 13 traffic conditions, such as: TVL A with E, TVL B with D, TVL B with E, TVL C with C, TVL C with D, TVL C with E, TVL D with C, TVL D with D, TVL D with E, TVL E with B, TVL E with C, TVL E with D, and TVL E with E. PCE values and TVL duration have a negative exponential relationship, in a few scenarios as shown in Figure 5. Under these situations, queue death rates caused by both the slow passenger car and the truck are lower than queue birth rate, so both types of slow vehicles cause an infinite queue where queue length is related to time. Therefore, the value of the PCE factor is a function of time. Several interesting observations are discovered under these situations: (1) PCE decreases as congestion duration increases, which shows that trucks will have a decreasing impact on traffic with congestion duration increasing when passing is not an option for both trucks and passenger cars. (2) Decreasing rate in PCE follows a negative exponential function, it decreases with greater speed at the beginning when congestion begins from the opposing direction. As congestion duration increases to certain value, the decreasing rate in PCE begins to drop and become very smooth or flat. (3) The TVL duration bench mark or inflection point for PCE decreasing rate approach to zero varies from 20 to 30 minutes and few

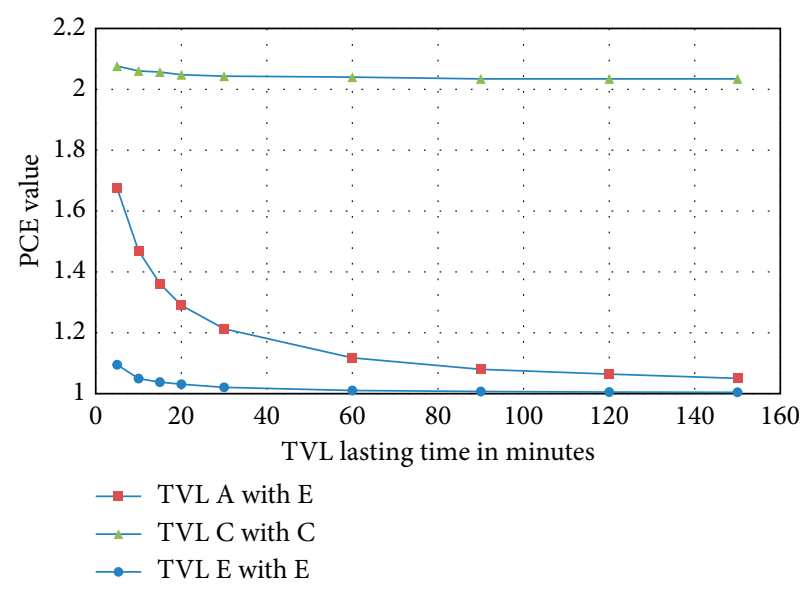

FIGURE 5: Negative exponential relationship.

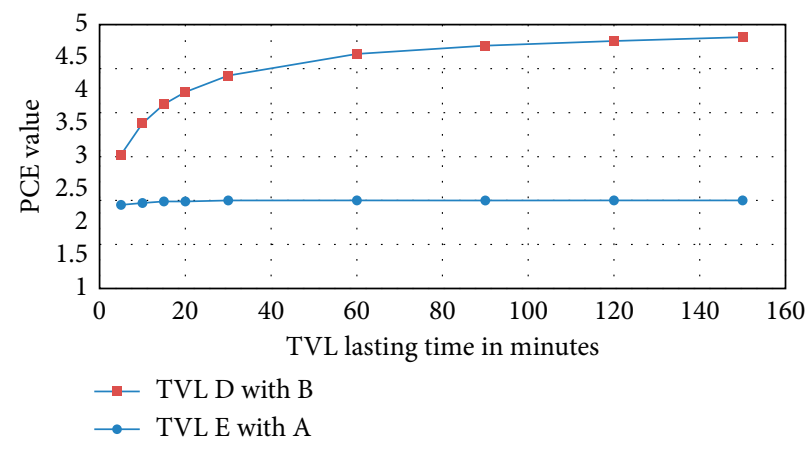

FIGURE 6: Logarithmic relationships.

(TVL A with E) begins at one hour. This means that PCE tends to stabilize and approach to a fixed floor value when severe congestion lasts for about 20-30 minutes. (4) Overall, there is no obvious fluctuation of PCE values when TVL duration increases from 0 to 150 minutes. The most noticeable change of PCE values is observed when traffic condition is under TVL A with E, where the PCE value decreases from 1.67 to 1.05. However, the most notable change is less than one unit. In addition, in this category, PCE values are relatively low, ranging from 2.1 to 1 , and most groups of PCE values are concentrated below 1.5. Only when it comes to TVL C with $\mathrm{C}$, the PCE value reaches 2.0.

3.3. Logarithmic Relationship. Under TVL D with B and TVL E and A, PCE values and TVL lasting time have a logarithmic relationship, as shown in Figure 6. Under these situations, queue lengths caused by both truck and passenger car increase with prevailing TVL duration. Several interesting observations are discovered under these situations: (1) PCE increases as congestion duration increases which shows that trucks will have increasing impact on traffic as congestion duration increases. (2) The increasing rate in PCE follows a logarithmic function. It increases at a decreasing rate. As congestion duration increases to a certain value, the increasing rate in PCE begins to drop and become very smooth or flat. (3) The TVL duration bench mark or inflection point for PCE 
increasing rate approach to zero varies from 20 to 30 minutes. This means that PCE tends to stabilize and approach a fixed ceiling value when severe congestion lasts for about 20-30 minutes, and one hour later the PCE stabilized at the fixed value. (4) PCE values of TVL D with B have a relatively evident fluctuation that increases from 3.0 to 4.8. PCE factors under TVL D with B tend to stabilize at 5.0. Under TVL of E with A, PCE values tend to stabilize around value of 2.3.

3.4. Nonrelated with Time. under TVL A with A, TVL A with $B$, TVL A with C, TVL B with A, TVL B with B, TVL C with A, and TVL $C$ with $B, P C E$ values do not vary with TVL duration. It is observed that under these situations, TVL of both lanes are good, and driving condition is comfortable with less traffic. Therefore, slow vehicles only affect a small amount of traffic, and cause queues with less than five vehicles. Therefore, the PCE value mostly depends on the headway model, which is mostly about trucks physical characteristics.

As shown in Table 1, PCE factor values are presented under all TVL combinations. From Table 1, it is concluded that (1) for a specific TVL in analysis direction, PCEs tends to increase and then decrease with the change of TVL in opposite direction changing from A to E. (2) PCE increase happens at less congested opposing lane condition when analysis direction of TVL is better. For example, for analysis direction of TVL A, PCE increases from 2.2 to 2.35 when opposing lane TVL begins at D. However, for analysis direction of TVL B, PCE begins to increase from 2.2 to 2.53 when opposing lane TVL starts at C. (3) unbalanced flow is a factor that effects on PCE. However, the TVL in both direction matters too. For example, the very unbalanced situation such as TVL A with $\mathrm{D}$ has a PCE of 2.35, however, a less Unbalanced situation such as TVL B with C has a higher PCE value of 2.53. (4) TVL duration has a great impact on PCEs. It is observed that high PCE values (higher than 3) happen more frequently when congestion duration reaches more than 30 minutes, and when the TVL of each lane is different.

Comparing PCE values in Table 1 and the value of less than 2.0 given by HCM 2010, the values presented by HCM can be either underestimated or overestimated depending on the TVLs of both directions. For example, when opposing lane is really congested (TVL E), the PCE values presented in this paper all approach 1 which is lower than the value presented by HCM 2010 [17]. When the analyzed lane is under TVL B with opposing lane of TVL C, PCE values computed in this paper are all much higher than 2, especially when TVL duration gets long.

\section{Research Limitations and Future Study}

The model developed in this paper is an analytical method. All criteria in the model should be described and calculated by mathematical methods. Several factors are known having impacts on PCE factors and indicated by previous researchers. Weather related factors such as windy weather, snow, hail, and even flooded road caused by heavy rain may impact PCE factors because large vehicles are difficult to maneuver in bad weather where quick movement or lane changes are necessary.
TABLE 1: PCE factor values.

\begin{tabular}{|c|c|c|c|c|c|c|c|}
\hline \multirow{4}{*}{$\begin{array}{l}\text { Opposite } \\
\text { lane TVL } \\
\mathrm{A}\end{array}$} & \multicolumn{7}{|c|}{ PCE factor value } \\
\hline & \multirow{2}{*}{$\begin{array}{c}\text { Analysis } \\
\text { lane } \\
\text { TVL } \\
\text { A }\end{array}$} & \multicolumn{6}{|c|}{ Opposite lane TVL lasting time } \\
\hline & & $5 \mathrm{~min}$ & $15 \mathrm{~min}$ & $30 \mathrm{~min}$ & $60 \mathrm{~min}$ & $90 \mathrm{~min}$ & $120 \mathrm{mir}$ \\
\hline & & 2.2 & 2.2 & 2.2 & 2.2 & 2.2 & 2.2 \\
\hline $\mathrm{B}$ & & 2.2 & 2.2 & 2.2 & 2.2 & 2.2 & 2.2 \\
\hline $\mathrm{C}$ & & 2.2 & 2.2 & 2.2 & 2.2 & 2.2 & 2.2 \\
\hline $\mathrm{D}$ & & 2.35 & 2.60 & 2.88 & 4.16 & 5.43 & 6.71 \\
\hline \multirow[t]{2}{*}{$\underline{E}$} & & 1.67 & 1.36 & 1.21 & 1.11 & 1.08 & 1.06 \\
\hline & B & & & & & & \\
\hline $\mathrm{A}$ & & 2.2 & 2.2 & 2.2 & 2.2 & 2.2 & 2.2 \\
\hline $\mathrm{B}$ & & 2.2 & 2.2 & 2.2 & 2.2 & 2.2 & 2.2 \\
\hline $\mathrm{C}$ & & 2.53 & 3.03 & 3.46 & 3.89 & 5.47 & 7.05 \\
\hline $\mathrm{D}$ & & 1.89 & 1.76 & 1.70 & 1.67 & 1.65 & 1.65 \\
\hline \multirow[t]{2}{*}{$\underline{E}$} & & 1.37 & 1.16 & 1.08 & 1.04 & 1.03 & 1.02 \\
\hline & $\mathrm{C}$ & & & & & & \\
\hline A & & 2.6 & 2.6 & 2.6 & 2.6 & 2.6 & 2.6 \\
\hline B & & 3.1 & 3.1 & 3.1 & 3.1 & 3.1 & 3.1 \\
\hline $\mathrm{C}$ & & 2.08 & 2.06 & 2.05 & 2.04 & 2.04 & 2.04 \\
\hline $\mathrm{D}$ & & 1.53 & 1.39 & 1.35 & 1.32 & 1.31 & 1.31 \\
\hline \multirow[t]{2}{*}{$\mathrm{E}$} & & 1.25 & 1.10 & 1.05 & 1.03 & 1.02 & 1.01 \\
\hline & $\mathrm{D}$ & & & & & & \\
\hline A & & 2.10 & 2.09 & 2.07 & 2.06 & 2.56 & 3.06 \\
\hline B & & 3.02 & 3.78 & 4.23 & 4.56 & 4.69 & 4.76 \\
\hline $\mathrm{C}$ & & 1.45 & 1.34 & 1.30 & 1.29 & 1.28 & 1.28 \\
\hline $\mathrm{D}$ & & 1.29 & 1.19 & 1.16 & 1.14 & 1.14 & 1.13 \\
\hline \multirow[t]{2}{*}{$\underline{\mathrm{E}}$} & & 1.16 & 1.06 & 1.03 & 1.02 & 1.01 & 1.01 \\
\hline & $\mathrm{E}$ & & & & & & \\
\hline A & & 2.26 & 2.31 & 2.33 & 2.34 & 2.34 & 2.34 \\
\hline B & & 1.66 & 1.60 & 1.58 & 1.57 & 1.56 & 1.56 \\
\hline $\mathrm{C}$ & & 1.26 & 1.19 & 1.17 & 1.16 & 1.16 & 1.16 \\
\hline $\mathrm{D}$ & & 1.20 & 1.14 & 1.12 & 1.11 & 1.11 & 1.10 \\
\hline $\mathrm{E}$ & & 1.10 & 1.03 & 1.02 & 1.01 & 1.01 & 1.00 \\
\hline
\end{tabular}

Their size also makes it impossible to come to a sudden stop. Moreover, those factors will also have impact on the lane congestion level detections [25-27], which in turn may also have impact on PCEs. However, due to the inability to mathematically model the weather related factors, they were ignored from this analytical study.

Wiseman [25-27] indicated in their research that light snow and heavy snow will have an impact on detecting congestion accuracy differently, light snow may over estimate congestion level while under heavy snow situation the congestion level can be estimated accurately. Considering if the LOS is collected under automatic monitoring system with such errors, then the PCE could be under-estimated. For example, if the actual LOSs are B for both analysis and opposing lanes, then the PCE factors should be 2.2, however, if the LOSs were overestimated under light snow weather as $\mathrm{C}$ for both lanes, then the PCE will be estimated as 2.08 even for a short period situation.

Lane width, and shoulder width which has known impact on PCE were also assumed as an average default value in this 
study. Moreover, only level terrain is assumed and no curvature and grade are considered. However, all these factors can be reflected in traffic performance. Thus, to calculate condition that is not included in this paper, traffic performance can be changed under certain conditions. For example, to study a highway segment with a narrower lane width, average travel speed can be reset with lower values.

As summarized above, this set of PCE values is not applicable for all situations. Further study is needed to complete PCE values to account for additional factors when data is available, such as vehicle types, terrain types which can be explained by the mathematical models. For example, if curvature information is available for a specific road segment, two factors can be affected: (1) truck and passenger car travel speeds and (2) passing sight distance. In general expectations, the narrower lane and shoulder width, and/or terrains with higher curvatures and grades, the higher PCE values are expected due to the bigger impact on truck operations compare to passenger car operations under those situations.

To account factors which are not easily modeled such as weather related factors, simulation-based method is recommended.

\section{Conclusions}

PCE impacts of traffic congestion levels and their durations of both lanes for a TLTW highway is under-researched in the literature. This research studied the impacts and developed a new recommended PCE values for heavy trucks on a TWTL highway with passing allowed. The following can be concluded for PCE on a two-way-two-lane highway:

(1) Traffic congestion levels on both two lanes have significant impacts on PCE values and the impact is not independently existent. The same congestion level of analysis lane has significantly different values if the opposite lane congestion level varies.

(2) Unbalanced traffic congestion levels have significant impact on PCE values. When analysis lane is at $\mathrm{A}$ or $\mathrm{B}$ congestion levels, the PCE increases first and then decrease with the unbalance traffic level increases. When analysis lane is at C or D congestion levels, the PCE increases first and then decreases with increased unbalanced traffic levels caused by improving opposite lane congestion levels but the PCE continuously decreases with increased unbalanced traffic levels caused by worsened opposite lane congestion levels.

(3) Traffic congestion level duration time has significant impact on PCE values. Duration time has less impact on PCE when traffic is balanced, in other words, PCEs maintain at the same levels when traffic congestions levels are the same for both lanes. However, duration time has higher impact on PCEs when traffic are unbalanced. For example, when traffic congestion level is A for analysis lane and opposite lane is at D level, the PCEs increase from 2.35 to 6.71 with the duration time increases from 5 to 120 minutes.
(4) The current PCE values adopted by HCM 2010 can significantly under-estimate HV's impact on traffic. For example, when traffic congestion level is B for analysis lane and opposite lane is at level $\mathrm{C}$ and if level $\mathrm{C}$ maintained for one and half hour, the PCE factor can be as high as 7.05 .

\section{Data Availabitlity}

The comma-separated values (CSV) data used to support the findings of this study are available from the corresponding author upon request.

\section{Conflicts of Interest}

The research work has no conflict of interest with the funding resource to support the work and no other possible conflict of interest in the manuscript.

\section{Acknowledgments}

The work presented in this paper was conducted with support from North Dakota State University and the Mountain-Plains Consortium, a University Transportation Center funded by the U.S. Department of Transportation. The contents of this paper reflect the views of the authors, who are responsible for the facts and accuracy of the information presented.

\section{References}

[1] M. V. Chitturi and R. F. Benekohal, Methodology for Development of Delay-Based Passenger Car: Equivalents of Heavy Vehicles in Work Zones, VDM Verlag Dr. Müller Aktiengesellschaft \& Co. KG, 2010.

[2] H. Nassiri, S. Tabatabaie, and S. Sahebi, "Delay-based passenger car equivalents at signalized intersections in Iran," Traffic of Transportation, vol. 29, no. 2, pp. 135-142, 2017.

[3] R. A. Krammes and K. W. Crowley, "Passenger car equivalents for trucks on level freeway segments," Transportation Research Record, vol. 1091, pp. 10-17, 2008.

[4] J. Craus, P. Abishai, and G. Itzhak, "A revised method for thedetermination of passenger car equivalencies," Transportation Research Part A: General, vol. 14, no. 4, pp. 241-246, 1980.

[5] E. Keller and J. Saklas, "Passenger car equivalents from network simulation," Journal of Transportation Engineering, vol. 110, no. 4, pp. 397-411, 1984.

[6] Transportation Research Board, Highway Capacity Manual 1965, TRB National Research Council, Washington, DC, USA, 1965.

[7] A. D. St. John, "Nonlinear truck factor for two-lane highways," in 55th Annual Meeting of the Transportation Research Board, Transportation Research Board Washington, DC, USA, 1976.

[8] E. Linzer, R. Roess, and W. McShane, Effect of Trucks, Buses, and Recreational Vehicles on Freeway Capacity and Service Volume, Transportation Research Board, Washington, DC, USA pp. 17-26, 1979.

[9] L. Elefteriadou, D. Torbic, and N. Webster, "Development of passenger car equivalents for freeways, two-lane highways, 
and arterials," Transportation Research Record: Journal of the Transportation Research Board, vol. 1572, no. 1, pp. 51-58, 1997.

[10] M. Van Aerde and S. Yagar, Capacity, Speed, and Platooning Vehicle Equivalents for Two-Lane Rural Highways, Transportation Research Board, Washington, DC, USA, 1984.

[11] N. Webster and L. Elefteriadou, "A simulation study of truck passenger car equivalents PC on basic freeway sections," Transportation Research Part B: Methodology, vol. 33, no. 5, pp. 323-336, 1999.

[12] Transportation Research Board, Highway Capacity Manual 2000, TRB National Research Council, Washington, DC, USA, 2000.

[13] A. Al-Kaisy, Y. Jung, and H. Rakha, "Developing passenger car equivalency factors for heavy vehicles during congestion," Journal of Transportation Engineering, vol. 131, no. 7, pp. 514-523, 2005.

[14] New England Section ITE Technical Committee, "Evaluation of average effective vehicle length queue," http://neite.org/ Documents/Technical/ITE\%20Tech\%20Comm\%20Queue\%20 Study-Final.pdf.

[15] Transportation Research Board, Highway Capacity Manual 1985, TRB National Research Council, Washington, DC, USA, 1985.

[16] A. Polus, J. Craus, and I. Grinberg, "Downgrade speed characteristics of heavy vehicles," Transportation Engineering Journal of ASCE, vol. 107, no. 2, pp. 143-152, 1981.

[17] Transportation Research Board, Highway Capacity Manual 2010, TRB National Research Council, Washington, DC, USA, 2010.

[18] S. H. Wolfgang, E. K. Louis, and R. M. William, Transportation and Traffic Engineering Handbook, Prentice-Hall Inc., New Jersey, NJ, USA, 1982.

[19] B. U. Physics, "Relative velocity" 1999, http://physics. bu.edu/ duffy/py105/RelativeV.html.

[20] D. L. Gerlough and M. J. Huber, Traffic Flow Theory, Transportation Research Board Special Report, Washington DC, USA, 1976.

[21] H. J. Van Zuylen and F. Viti, "Queues at controlled intersections: the old theory revised," in Proceedings of the IEEE-ITSC'06 Conference, IEEE, Toronto, Canada, 2006.

[22] L. Vasconce, A. B. Silva, Alvaro Seco, and J. Silva, "Estimating the parameters of Cowan's M3 headway distribution for roundabout capacity analyses," The Baltic Journal of Road and Bridge Engineering, vol. 7, no. 4, pp. 261-268, 2012.

[23] Transportation Engineering Online Lab Manual, "Geometric design theory and concepts," 2003, https://www.webpages. uidaho.edu/niatt_labmanual/Chapters/geometricdesign/ theoryandconcepts/PassingSightDistance.htm.

[24] Commercial Transport Act, "Commercial transport regulation," 2011, http://www.bclaws.ca/Recon/document/ID/ freeside/30_78.

[25] Y. Wiseman, "Real-time monitoring of traffic congestions," in IEEE International Conference on Electro Information Technology (EIT 2017), IEEE, Lincoln, NE, USA, 2017.

[26] Y. Wiseman, "Computerized traffic congestion detection system," The International Journal of Transportation and Logistics Management, vol. 1, no. 1, pp. 1-8, 2017.

[27] Y. Wiseman, "Tool for online observing of traffic congestions," The International Journal of Control and Automation, vol. 10, no. 6, pp. 27-34, 2017. 


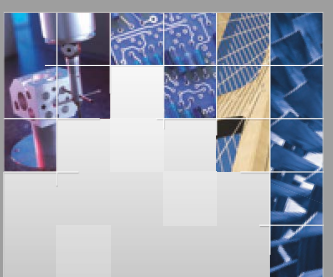

\section{Enfincering}
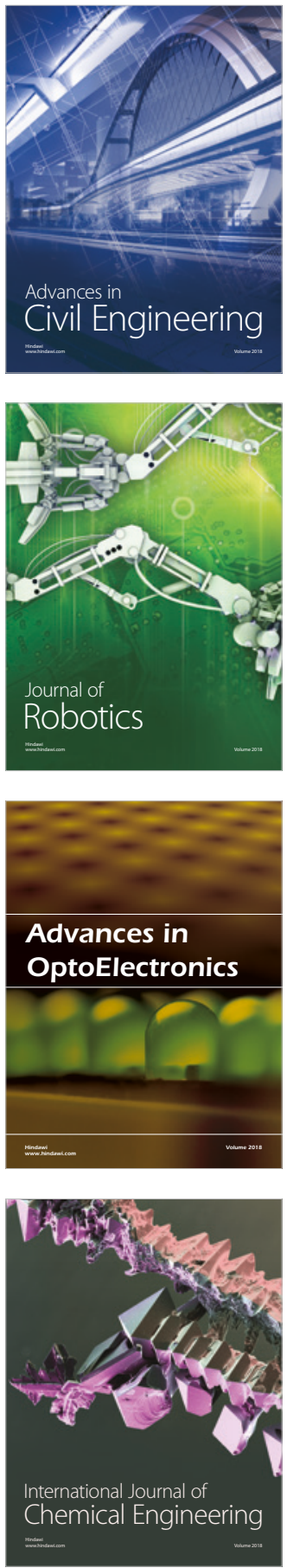

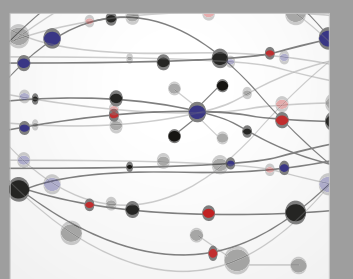

\section{Rotating \\ Machinery}

The Scientific World Journal

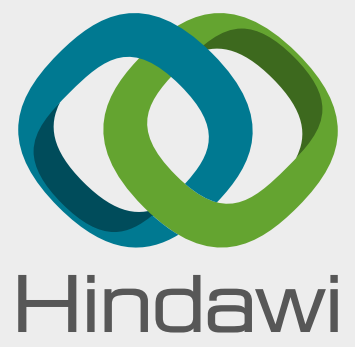

Submit your manuscripts at

www.hindawi.com
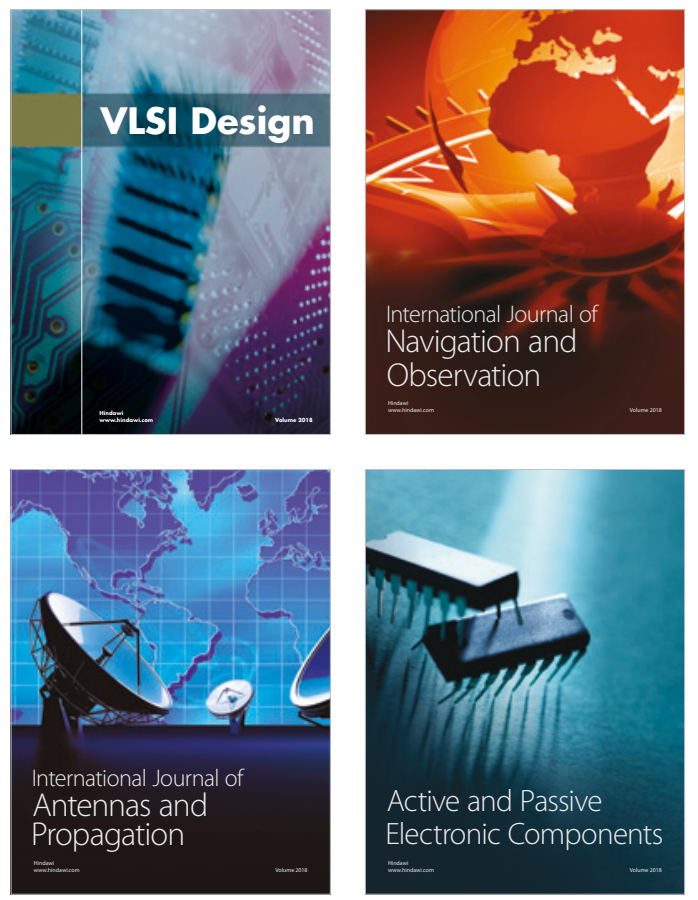
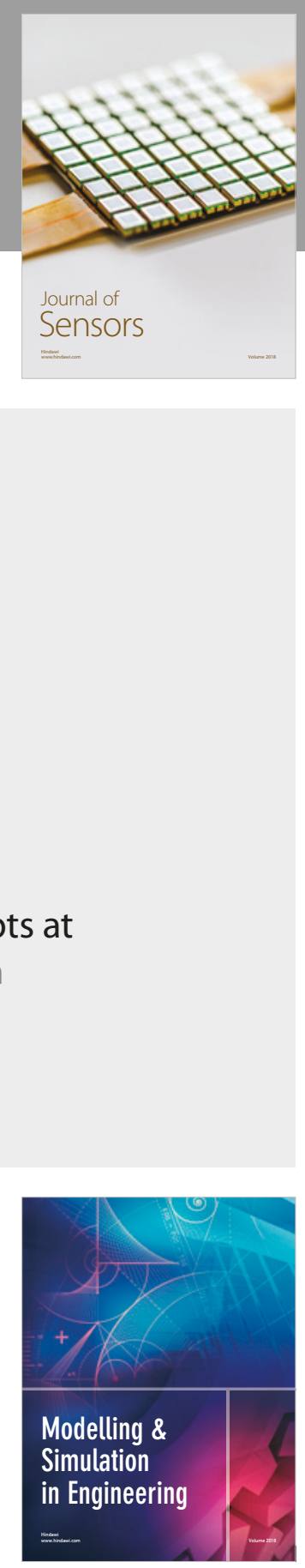

\section{Advances \\ Multimedia}
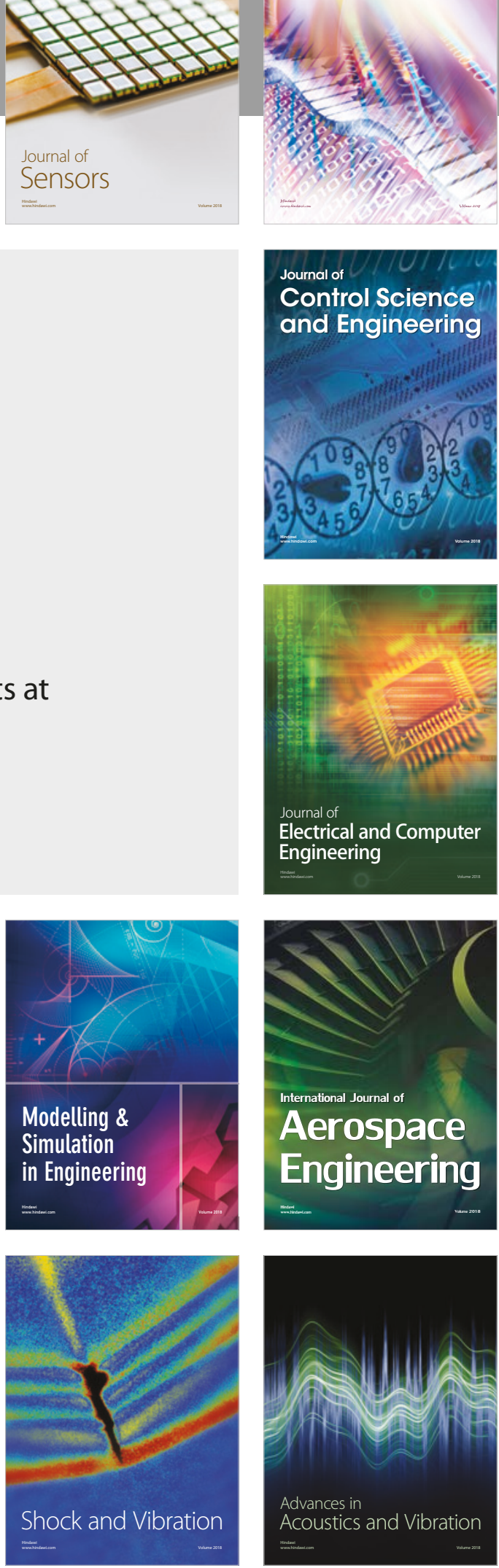